\title{
On provability logics with linearly ordered modalities
}

\author{
Lev D. Beklemishev* ${ }^{*}$ David Fernández-Duque ${ }^{\dagger}$ Joost J. Joosten ${ }^{\ddagger}$
}

September 27, 2018

\begin{abstract}
We introduce the logics $\mathrm{GLP}_{\Lambda}$, a generalization of Japaridze's polymodal provability logic $\mathrm{GLP}_{\omega}$ where $\Lambda$ is any linearly ordered set representing a hierarchy of provability operators of increasing strength.

We shall provide a reduction of these logics to $\mathrm{GLP}_{\omega}$ yielding among other things a finitary proof of the normal form theorem for the variablefree fragment of $\mathrm{GLP}_{\Lambda}$ and the decidability of $\mathrm{GLP}_{\Lambda}$ for recursive orderings $\Lambda$. Further, we give a restricted axiomatization of the variable-free fragment of $\mathrm{GLP}_{\Lambda}$.
\end{abstract}

\section{Introduction}

The provability logic $\mathrm{GLP}_{\Lambda}$ with transfinitely many modalities $\langle\alpha\rangle$, for all ordinals $\alpha<\Lambda$, generalizes the well-known provability logic GLP denoted $\mathrm{GLP}_{\omega}$ in this paper [21, 9]. The logic $\mathrm{GLP}_{\omega}$ has been used to carry out a proof-theoretic analysis of Peano Arithmetic and related theories using the approach of provability algebras initiated in [3]. A natural next class of theories to analyze with this new approach are predicative theories such as the second order theories of iterated arithmetical comprehension and $\mathrm{ATR}_{0}$. The first necessary step towards analyzing predicative theories with provability algebras was made in [4] where logics $\mathrm{GLP}_{\Lambda}$, for an arbitrary ordinal $\Lambda$, were introduced and it was shown that the variable-free fragments of these logics yield a natural ordinal notation system up to the ordinal $\Gamma_{0}$.

\footnotetext{
${ }^{*}$ V.A. Steklov Mathematical Institute, RAS; Moscow M.V. Lomonosov State University; NRU Higher School of Economics; bekl@mi.ras.ru

${ }^{\dagger}$ Group for Logic, Language and Computation, University of Seville, dfduque@us.es

${ }^{\ddagger}$ Dept. Lògica, Història i Filosofia de la Ciència, Universitat de Barcelona, jjoosten@ub.edu
} 
Assuming an ordinal $\Lambda$ to be represented, ordinals of a possibly larger class can be denoted by modal formulas (called words or worms) of the form

$$
\left\langle\alpha_{1}\right\rangle\left\langle\alpha_{2}\right\rangle \ldots\left\langle\alpha_{n}\right\rangle \top
$$

where $\alpha_{i}<\Lambda$, identified modulo provable equivalence in $\mathrm{GLP}_{\Lambda}$. The ordering between two words $A$ and $B$ is naturally defined by

$$
A<_{0} B \Longleftrightarrow \mathrm{GLP}_{\Lambda} \vdash B \rightarrow\langle 0\rangle A .
$$

It was shown that this ordering is a well-ordering, and basic formulas for the computation of the order types of its initial segments in terms of Veblen ordinal functions were found in 4 .

Since then, the logics $\mathrm{GLP}_{\Lambda}$ and their ordinal notation systems have been studied in much more detail (see [16, 13, 14]). Most importantly, suitable Kripke models for the variable-free fragment of $\mathrm{GLP}_{\Lambda}$ generalizing the so-called Ignatiev model for $\mathrm{GLP}_{\omega}$ [20] have been developed. Also, the completeness of $\mathrm{GLP}_{\Lambda}$ w.r.t. topological semantics has been proved [1, 12]. Some of these papers used the normal form results from [4].

Sections 4 and 5 of the present paper is in many respects a 'recasting' of the part of [4] devoted to the normal forms for the variable-free fragment of GLP $_{\Lambda}$ and to its axiomatizations. The main reason to have such a recasting is that the exposition in [4] was at some places overly sketchy, to the extent that some parts of the arguments were only hinted at. The main such omission was the proof of the fact that the ordering $<_{0}$ on words was irreflexive, or equivalently the fact that any individual word was consistent with $G L P_{\Lambda}$. Modulo this claim, the rest of the arguments in the paper were purely syntactical or dealt with ordinal computations. For this consistency result one would naturally use some kind of semantics, which were not available at the time for $\Lambda>\omega$ (but see [13]).

Another reason for having a recast of parts of [4] is that the authors of [13] needed certain results -in particular, Corollaries 5.11 and 5.12 of the current paper - that follow from the line of reasoning presented in [4]. However, a proof of these corollaries could not be given without revisiting and sharpening various results from [4].

Moreover, it was remarked in [4] that the irreflexivity of $<_{0}$ follows, for example, from any arithmetically sound interpretation of $G L P_{\Lambda}$ w.r.t. a sequence of strong provability predicates. Indeed, the existence of such interpretations was obvious at least for constructive ordinals $\Lambda$. On the other hand, a proof appealing to such an interpretation is necessarily based on the assumption of soundness of a fairly strong extension of Peano Arithmetic and 
thus cannot be formalized in Peano Arithmetic itself. For proof-theoretic applications we would like to have an ordering representation whose elementary properties such as irreflexivity are provable by finitary means (e.g., in Primitive Recursive Arithmetic). Alternative proofs based on the use of Ignatiev-like models or topological models for $\mathrm{GLP}_{\Lambda}$ suffer from the same drawback.

In this paper we remedy this situation and provide a different purely modal finitary proof of irreflexivity based on a reduction of $\mathrm{GLP}_{\Lambda}$ to $\mathrm{GLP}_{\omega}$, for which such a finitary proof is known [2]. We also prove the conservativity of $\mathrm{GLP}_{\Lambda}$ over any of its restrictions to a subset of modalities. This reduction uses the methods of [6].

The exposition of the normal form theorem for variable-free formulas in $G L P_{\Lambda}$ in this paper is also slightly different from the one in [3, 4. Namely, the normal forms are defined in a 'positive' way, which helps, in particular, to eliminate the assumption of irreflexivity at some places where it is not necessary. Finally, we provide a more restricted axiomatization of the variable-free fragment of $\mathrm{GLP}_{\Lambda}$ than the one in [4].

An additional novelty of this paper is that the results can be stated and proved in a more general context of logics with linearly ordered sets of modalities. Thus, from the outset we introduce and work with a generalization of GLP $_{\Lambda}$ to the case when $\Lambda$ is an arbitrary, not necessarily well-founded, linear ordering. So far, proof-theoretic interpretations of such logics have not been investigated; however it seems likely that they can appear, for example, in the study of progressions of theories defined along recursive linear orderings without infinite hyperarithmetical descending sequences (see, e.g., [11]).

\section{The logic $\mathrm{GLP}_{\Lambda}$ and its fragments}

In this section we shall introduce the formal systems that we will study throughout the paper. Our logics depend on a parameter, usually denoted $\Lambda$, which is a linear order of the form $\langle|\Lambda|,<\rangle$. They then contain a modality $[\alpha]$ for each $\alpha \in|\Lambda|$. In analogy to the set-theoretic treatment of ordinals, we will identify $\Lambda$ with an upper bound for its elements and often write $\alpha<\Lambda$ instead of $\alpha \in|\Lambda|$; elements of $|\Lambda|$ will sometimes be called modals. Note, however, that unlike previous studies of $\mathrm{GLP}_{\Lambda}$, we allow for $\Lambda$ to be an arbitrary linear order.

We will also introduce some important fragments of $\mathrm{GLP}_{\Lambda}$. These fragments are easier to work with from a technical point of view, yet they already contain much of the crucial information about the full logic, as we shall see. 


\subsection{The logics $\mathrm{GLP}_{\Lambda}$}

The full language $\mathcal{L}_{\Lambda}$ is built from propositional variables in a countably infinite set $\mathbb{P}$ and the constant $\top$ together with the Boolean connectives $\neg, \wedge$ and a unary modal operator $[\alpha]$ for each $\alpha \in \Lambda$. As is customary, other Boolean operators may be defined in the standard way and we write $\langle\alpha\rangle$ as a shorthand for $\neg[\alpha] \neg$.

We will use $\bmod \phi$ to denote the set of elements of $|\Lambda|$ appearing in $\phi$ and $\max \phi$ to be the maximum of these modals. We also use $l(\phi)$ to denote the length of $\phi$, defined in a standard way, and $w(\phi)$ to be its width, that is, the number of modals appearing in $\phi$.

Definition $2.1\left(\mathrm{GLP}_{\Lambda}\right)$. Given a linear order $\Lambda=\langle|\Lambda|,<\rangle, \mathrm{GLP}_{\Lambda}$ is the logic over $\mathcal{L}_{\Lambda}$ given by the following rules and axioms:

- All substitution instances of propositional tautologies,

- For all $\alpha, \beta \in|\Lambda|$ and formulas $\chi, \psi \in \mathcal{L}_{\Lambda}$,

$$
\begin{array}{lll}
\text { (i) } & {[\alpha](\chi \rightarrow \psi) \rightarrow([\alpha] \chi \rightarrow[\alpha] \psi)} & \\
\text { (ii) } & {[\alpha]([\alpha] \chi \rightarrow \chi) \rightarrow[\alpha] \chi} & \\
\text { (iii) } & {[\alpha] \chi \rightarrow[\beta][\alpha] \chi} & \text { for } \alpha \leq \beta \\
\text { (iv) } & \langle\alpha\rangle \chi \rightarrow[\beta]\langle\alpha\rangle \chi & \text { for } \alpha<\beta, \\
\text { (v) } & {[\alpha] \chi \rightarrow[\beta] \chi} & \text { for } \alpha \leq \beta .
\end{array}
$$

- Modus Ponens and the necessitation rule $\frac{\chi}{[\alpha] \chi}$ for each modality $\alpha \in$ $|\Lambda|$.

This definition contains certain redundancies: Axiom (iii) is clearly derivable in presence of the others, and necessitation for 0 would suffice given Axiom $(v)$. However, it will be convenient to state these principles separately.

\subsection{Kripke semantics}

Kripke models give us a transparent and convenient interpretation for many modal logics. A Kripke frame is a structure $\mathfrak{F}=\left\langle W,\left\langle R_{\lambda}\right\rangle_{\lambda<\Lambda}\right\rangle$, where $W$ is a set and $\left\langle R_{\lambda}\right\rangle_{\lambda<\Lambda}$ a family of binary relations on $W$. A valuation on $\mathfrak{F}$ is a function $\llbracket \cdot \rrbracket: \mathcal{L}_{\Lambda} \rightarrow \mathcal{P}(W)$ such that

$$
\begin{aligned}
& \llbracket \perp \rrbracket \quad=\varnothing \quad \llbracket \neg \phi \rrbracket \quad=\quad W \backslash \llbracket \phi \rrbracket \\
& \llbracket \phi \wedge \psi \rrbracket=\llbracket \phi \rrbracket \cap \llbracket \psi \rrbracket \quad \llbracket\langle\lambda\rangle \phi \rrbracket=R_{\lambda}^{-1} \llbracket \phi \rrbracket .
\end{aligned}
$$




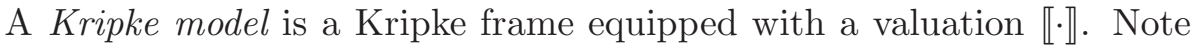
that propositional variables may be assigned arbitrary subsets of $W$. Often we will write $\langle\mathfrak{F}, \llbracket \cdot \rrbracket\rangle, x \Vdash \psi$ instead of $x \in \llbracket \psi \rrbracket$ or even just $x \Vdash \psi$ if the context allows us to. As usual, $\phi$ is satisfied on $\langle\mathfrak{F}, \llbracket \cdot \rrbracket\rangle$ if $\llbracket \phi \rrbracket \neq \varnothing$, and valid on $\langle\mathfrak{F}, \llbracket \cdot \rrbracket\rangle$ if $\llbracket \phi \rrbracket=W$. The latter case shall be denoted by $\langle\mathfrak{F}, \llbracket \cdot \rrbracket\rangle \models \psi$.

We shall also use the notion of frame validity in that $\mathfrak{F}, x \models \psi$ denotes that $\langle\mathfrak{F}, \llbracket \cdot \rrbracket\rangle, x \Vdash \psi$ for any valuation $\llbracket \cdot \rrbracket$. Likewise, $\mathfrak{F} \models \psi$ denotes that $\mathfrak{F}, x \models \psi$ for all $x$ in $\mathfrak{F}$.

$\mathrm{GLP}_{\Lambda}$ has no non-trivial Kripke models, but its variable-free or closed fragment (defined below) does [20]. We will use a sublogic $J_{\Lambda}$ of $\mathrm{GLP}_{\Lambda}$ that is sound and complete w.r.t. a suitable class of finite frames called $J$-frames. The logics $J_{\Lambda}$ can be obtained from the given axiomatization of $\mathrm{GLP}_{\Lambda}$ by replacing the monotonicity axiom schema (v) by the following schema (derivable in $\mathrm{GLP}_{\Lambda}$ ):

$$
[\alpha] \phi \rightarrow[\alpha][\beta] \phi, \text { for } \alpha \leq \beta .
$$

This system has been introduced in [6] just for the language $\mathcal{L}_{\omega}$. Although it is easy to see that the Kripke model completeness theorem for $J_{\omega}$ proved in [6] holds more generally, we will actually use it only for the logic $J_{\omega}$.

A Kripke frame is called a $J_{\Lambda}$-frame if, for all $\beta<\alpha<\Lambda$,

- $R_{\alpha}$ is a conversely well-founded, transitive ordering relation on $W$;

- $\forall x, y\left(x R_{\alpha} y \Rightarrow \forall z\left(x R_{\beta} z \Leftrightarrow y R_{\beta} z\right)\right)$;

- $\forall x, y\left(x R_{\alpha} y \& y R_{\beta} z \Rightarrow x R_{\alpha} z\right)$.

A $\mathrm{J}_{\Lambda}$-frame is called finite if so is the set of its nodes $W$. A $\mathrm{J}_{\Lambda}$-model is a Kripke model based on a $J_{\Lambda}$-frame.

The following is proved in [6] for $\Lambda=\omega$, but holds more generally with the same proof.

\section{Proposition 2.2.}

1. If $\mathrm{J}_{\Lambda} \vdash \phi$ then $\phi$ is valid in all $J_{\Lambda}$-models;

2. If $\mathrm{J}_{\Lambda} \nvdash \phi$ then $\phi$ is not valid in some finite $J_{\Lambda}$-model.

\section{$2.3 \quad$ Fragments of $\mathrm{GLP}_{\Lambda}$}

There are two particular families of sublogics of $\mathrm{GLP}_{\Lambda}$ which we will focus on later. The first is the fragment without variables, which as we shall see is already quite expressive: 
Definition 2.3 (Closed fragment). We denote by $\mathcal{L}_{\Lambda}^{0}$ the sublanguage of $\mathcal{L}_{\Lambda}$ whose formulas do not contain propositional variables (only $\top$ ).

$\mathrm{GLP}_{\Lambda}^{0}$ denotes the intersection of $\mathrm{GLP}_{\Lambda}$ with $\mathcal{L}_{\Lambda}^{0}$.

That is, GLP ${ }_{\Lambda}^{0}$ is the set of provable formulas of $\mathrm{GLP}_{\Lambda}$ that do not contain any propositional variables. It is clear that any closed formula $\psi$ provable in $\mathrm{GLP}_{\Lambda}^{0}$ can also be proved using proofs and axioms without variables. For, given a proof $\pi$ of $\psi$, we can substitute $\top$ (or $\perp$ ) for the propositional variables that occur in $\pi$. After substitution we still have a proof of $\psi$.

The second fragment is the restriction to a subset of all modals, which is especially useful when this subset is finite.

Definition 2.4. For any subset $S \subseteq|\Lambda|$, let $\mathcal{L}_{S}$ denote the language with the set of modalities $\{[\xi]: \xi \in S\}$, and let $\mathrm{GLP}_{S}$ be the logid given by the restriction of the axioms and rules of $\mathrm{GLP}_{\Lambda}$ to $\mathcal{L}_{S}$.

As we shall see in Section 3 , any provable formula of $\mathcal{L}_{S}$ is also provable within $\mathrm{GLP}_{S}$. However, this is not as immediate as in the case of the closed fragment.

\section{Reduction of $\mathrm{GLP}_{\Lambda}$ to its finite fragments}

Here we show that $\mathrm{GLP}_{\Lambda}$ is conservative over any of its fragments obtained by restricting the language to a subset of its modalities.

Clearly, if $S$ is a set of ordinals, GLP $S$ is only notationally different from $\operatorname{GLP}_{\beta}$ where $\beta$ is the order type of $S$. More precisely, let $\xi_{\alpha}$ be the $\alpha$-th element of $S$ and let $\xi(\phi)$ denote the result of replacing in a formula $\phi$ (in the language $\mathcal{L}_{\beta}$ ) each modality $[\alpha]$ by $\left[\xi_{\alpha}\right]$. Similarly, let $\xi^{-1}(\psi)$ denote the inverse operation. Then the following lemma is obvious.

\section{Lemma 3.1.}

(i) $\mathrm{GLP}_{\beta} \vdash \phi$ iff $\mathrm{GLP}_{S} \vdash \xi(\phi)$;

(ii) $\mathrm{GLP}_{S} \vdash \psi$ iff $\mathrm{GLP}_{\beta} \vdash \xi^{-1}(\psi)$.

The conservation result is now stated as follows.

Theorem 3.2. Given a linear order $\Lambda, S \subseteq|\Lambda|$ and a formula $\phi$ in $\mathcal{L}_{S}$, $\mathrm{GLP}_{\Lambda} \vdash \phi$ iff GLP $\mathrm{G}_{S} \vdash \phi$.

\footnotetext{
${ }^{1}$ In principle we should include $\Lambda$ as a second parameter since these logics depend on the specific ordering, but we will let this be given by context.
} 
Proof. A proof will proceed in two steps. First, we prove the conservativity of $\mathrm{GLP}_{\omega}$ over any of its finite fragments. Secondly, we will use a purely syntactic argument to lift this result to arbitrary fragments of $\mathrm{GLP}_{\Lambda}$.

We are going to use the following standard reduction of $\mathrm{GLP}_{\omega}$ to $\mathrm{J}_{\omega}$ (see [6]). Let $\phi$ be a GLP $\omega_{\omega}$-formula, and let $\left\{\left[m_{i}\right] \phi_{i}: i<s\right\}$ be all the boxed subformulas of $\phi$ with $m_{i} \leq m_{j}$ whenever $i<j$. Denote:

$$
M^{+}(\phi):=M(\phi) \wedge \bigwedge_{i \leq m_{s}}[i] M(\phi)
$$

where

$$
M(\phi):=\bigwedge_{i<s} \bigwedge_{m_{i}<j \leq m_{s}}\left(\left[m_{i}\right] \phi_{i} \rightarrow[j] \phi_{i}\right) .
$$

The following result is proved in [6] using Kripke model techniques. Alternative proofs (using the topological and the arithmetical semantics, respectively) can be found in [8, 1]. The proof in [6] has the advantage of being formalizable in Elementary Arithmetic 2

Lemma 3.3. $\mathrm{GLP}_{\omega} \vdash \phi \Longleftrightarrow \mathrm{J}_{\omega} \vdash M^{+}(\phi) \rightarrow \phi$.

We are going to show here that the formula $M^{+}$can be replaced by a formally weaker one: $N^{+}(\phi):=N(\phi) \wedge \bigwedge_{i<s}\left[m_{i}\right] N(\phi)$ and

$$
N(\phi):=\bigwedge_{i<s} \bigwedge_{i<j<s}\left(\left[m_{i}\right] \phi_{i} \rightarrow\left[m_{j}\right] \phi_{i}\right)
$$

Notice that $N^{+}(\phi)$ is in the language of $\phi$.

Lemma 3.4. $\mathrm{GLP}_{\omega} \vdash \phi \Longleftrightarrow \mathrm{J}_{\omega} \vdash N^{+}(\phi) \rightarrow \phi$.

Proof. Suppose $\mathrm{J}_{\omega} \nvdash N^{+}(\phi) \rightarrow \phi$. Then there is a finite $\mathrm{J}_{\omega}$-model $\mathcal{W}$ with a node $r$ such that $\mathcal{W}, r \Vdash N^{+}(\phi)$ and $\mathcal{W}, r \nVdash \phi$. Replace each relation $R_{k}$ in $\mathcal{W}$ by $\emptyset$, for all $k \notin S:=\left\{m_{0}, \ldots, m_{s-1}\right\}$. The result is still a $J_{\omega}$-model (denoted $\mathcal{W}^{\prime}$ ), and the forcing of formulas in the language of $\phi$ is everywhere the same.

Finally, we observe that $M^{+}(\phi)$ is true at $r$. It is sufficient to show that each implication $\left[m_{i}\right] \phi_{i} \rightarrow[j] \phi_{i}$, for $m_{i}<j \leq m_{s}$, holds at each point $x \in \mathcal{W}^{\prime}$ reachable from $r$. We observe that such an $x$ is either $r$ itself or is reachable by one of the relations $R_{m_{i}}$, for $i<s$. Since $r \Vdash N(\phi) \wedge\left[m_{i}\right] N(\phi)$ we have $x \Vdash N(\phi)$. Hence, if $j \in S$ we have $x \Vdash\left[m_{i}\right] \phi_{i} \rightarrow[j] \phi_{i}$ as required. However, if $j \notin S$ the relation $R_{j}$ is empty, and thus $x \Vdash[j] \phi_{i}$ trivially. Thus, Lemma 3.4 follows from Lemma 3.3 .

\footnotetext{
${ }^{2}$ The formula $M(\phi)$ is misspelled in [6].
} 

$\mathcal{L}_{S}$.

For any $S \subseteq \omega$ let $\mathrm{J}_{S}$ denote the restriction of the logic $\mathrm{J}_{\omega}$ to the language

Lemma 3.5. For any formula $\phi$ in $\mathcal{L}_{S}, \mathrm{~J}_{\omega} \vdash \phi$ iff $\mathrm{J}_{S} \vdash \phi$.

Proof. Only the (only if) part needs to be proved. Assume $\mathrm{J}_{S} \nvdash \phi$. Consider any $\mathrm{J}_{\omega}$-model $\mathcal{W}$ in the restricted language $\mathcal{L}_{S}$ such that $\mathcal{W} \not \models \phi$. For each $i \notin S$, define a new relation $R_{i}$ on $\mathcal{W}$ by letting $R_{i}=\emptyset$. The expanded model $\mathcal{W}^{\prime}$ is a model of $J_{\omega}$ and $\mathcal{W}^{\prime} \not \models \phi$. Hence, $J_{\omega} \nvdash \phi$.

From Lemmas 3.4 and 3.5 we obtain the conservativity of GLP $\omega$ over its fragments.

Corollary 3.6. Let $S \subseteq \omega$ and $\phi$ be a formula in $\mathcal{L}_{S}$. Then $\mathrm{GLP}_{\omega} \vdash \phi$ iff $\mathrm{GLP}_{S} \vdash \phi$.

Now we turn to the general case and prove Theorem 3.2. Assume $\phi$ is in $\mathcal{L}_{S}$ and $\mathrm{GLP}_{\Lambda} \vdash \phi$. Let $R \subseteq|\Lambda|$ be the set of all modals occurring in the given derivation of $\phi$. The same derivation shows that $\mathrm{GLP}_{R} \vdash \phi$. Since $R$ is finite, we can assume it is enumerated by some function $\xi:\{0, \ldots, n-1\} \rightarrow R$. Let $\psi:=\xi^{-1}(\phi)$. By Lemma 3.1 we obtain $\mathrm{GLP}_{n} \vdash \psi$ and hence $\mathrm{GLP}_{\omega} \vdash \psi$.

Let $F$ be the set of modals occurring in $\phi$. Obviously, $F \subseteq R$ and $G:=\xi^{-1}(F) \subseteq \omega$. Therefore, by Corollary 3.6 GLP $\vdash \psi$. It follows that $\mathrm{GLP}_{\xi G} \vdash \xi(\psi)$, that is, GLP $\vdash$. Since $F \subseteq S$ we conclude that GLP $\vdash$. as required. This completes the proof of Theorem 3.2 .

For any formula $\phi$ let $\hat{\phi}$ denote $\xi^{-1}(\phi)$, where $\xi:\{0, \ldots, n-1\} \rightarrow F$ enumerates the set $F$ of all modals occurring in $\phi$. Applying Theorem 3.2 to $F$ we obtain the following corollary.

Corollary 3.7. For any $\phi, \mathrm{GLP}_{\Lambda} \vdash \phi$ iff $\mathrm{GLP}_{n} \vdash \hat{\phi}$ iff $\mathrm{GLP}_{\omega} \vdash \hat{\phi}$.

Proof. By Theorem 3.2, GLP ${ }_{\Lambda} \vdash \phi$ iff $\mathrm{GLP}_{F} \vdash \phi$, whereas by Lemma 3.1 the latter is equivalent to $\mathrm{GLP}_{n} \vdash \hat{\phi}$.

By this corollary, the logic $\mathrm{GLP}_{\Lambda}$ inherits many nice properties proved for $\mathrm{GLP}_{\omega}$. Let us state a few explicitly. Below, the corollaries follow directly from their counterparts as proven for $\mathrm{GLP}_{\omega}[20$, 7, 24].

Corollary 3.8. $\mathrm{GLP}_{\Lambda}$ is a decidable logic, provided $\Lambda$ has a recursive presentation. 
Corollary 3.9. GLP $\mathrm{GL}_{\Lambda}$ enjoys Craig interpolation: If $\psi(\vec{p}, \vec{q})$ and $\phi(\vec{q}, \vec{r})$ are $\mathcal{L}_{\Lambda}$-formulas with all variables among the distinct variables $\vec{p}, \vec{q}, \vec{r}$ with $\mathrm{GLP}_{\Lambda} \vdash \psi(\vec{p}, \vec{q}) \rightarrow \phi(\vec{q}, \vec{r})$, then there is some formula $\theta(\vec{q})$ whose variables are all among $\vec{q}$ such that

$$
\operatorname{GLP}_{\Lambda} \vdash(\psi(\vec{p}, \vec{q}) \rightarrow \theta(\vec{q})) \wedge(\theta(\vec{q}) \rightarrow \phi(\vec{q}, \vec{r})) .
$$

Corollary 3.10. $\mathrm{GLP}_{\Lambda}$ has unique fixpoints: Let $\psi(\vec{p}, q)$ be a formula of $\mathcal{L}_{\Lambda}$ where $q$ only occurs under the scope of a modality. Then, there exists some $\phi(\vec{p})$ such that $\psi(\vec{p}, q / \phi(\vec{p}))$ is $\mathrm{GLP}_{\Lambda}$-provably equivalent to $\phi(\vec{p})$. Moreover, this is provable within $\mathrm{GLP}_{\Lambda}$ itself:

$$
\mathrm{GLP}_{\Lambda} \vdash \odot(q \leftrightarrow \phi(\vec{p})) \leftrightarrow \odot(q \leftrightarrow \psi(\vec{p}, q))
$$

The standard variations of this theorem like unique solutions to simultaneous fixpoints equations also carry directly through to $\mathrm{GLP}_{\Lambda}$.

Corollary 3.11. $\mathrm{GLP}_{\Lambda}$ satisfies the uniform interpolation property: for any $\mathcal{L}_{\Lambda}$-formula $\psi(\vec{q}, \vec{r})$ with distinguished variables $\vec{q}$ there exists a uniform interpolant, that is, a formula $\phi(\vec{q})$ such that for any $\theta(\vec{q})$ we have

$$
\mathrm{GLP}_{\Lambda} \vdash \psi(\vec{q}, \vec{r}) \rightarrow \theta(\vec{q}) \quad \Longleftrightarrow \quad \mathrm{GLP}_{\Lambda} \vdash \phi(\vec{q}) \rightarrow \theta(\vec{q})
$$

\section{Worms and their normal forms}

In this section we study worms, or iterated consistency satements, which in a sense form the backbone of the logic $\mathrm{GLP}_{\Lambda}^{0}$ (recall that $\mathrm{GLP}_{\Lambda}^{0}$ is the fragment of $\mathrm{GLP}_{\Lambda}$ which contains no propositional variables). Worms directly code the ordinals needed for a proof-theoretic analysis of formal theories. Moreover, as we shall see, every closed formula of $\mathrm{GLP}_{\Lambda}^{0}$ can be written as a Boolean combination of worms.

Many of the results presented here appeared originally in [4]. The main difference is that we employ a different -but equivalent, as we shall seedefinition of normal forms on worms. We also include more details than in [4] and do not use the irreflexivity of the $<_{\alpha}$ relations.

Definition 4.1 (Worms). The set of words, or worms, is a subset of $\mathcal{L}_{\Lambda}^{0}$ denoted by $\mathbb{W}$ and is inductively defined as $\top \in \mathbb{W}$, and $A \in \mathbb{W} \Rightarrow\langle\alpha\rangle A \in$ $\mathbb{W}$ where $\alpha$ is a modal.

We write $\alpha \in A$ to indicate that $\alpha$ occurs somewhere in the word $A$. By $\mathbb{W}_{\alpha}$ we denote $\{A \in \mathbb{W} \mid \beta \in A \Rightarrow \beta \geq \alpha\}$. 
It is customary to identify a worm $A$ with the sequence of the modals in $A$. Thus, $\langle 0\rangle\langle 2\rangle \top$ will be associated with just 02 but we shall also employ any hybrid form like $\langle 0\rangle 2$, etc. We will associate $T$ with the empty sequence/word $\epsilon$. Worms owe their name to the heroic worm-battle, a variant of the Hydra battle (see [5]), but they may also be called words.

\subsection{Natural orderings on $\mathbb{W}_{\alpha}$}

On the set of worms one can define natural order relations.

Definition 4.2. For $A, B \in \mathbb{W}$ we define $A<_{\alpha} B: \Leftrightarrow \mathrm{GLP}_{\Lambda} \vdash B \rightarrow\langle\alpha\rangle A$.

It is clear by Axiom (iii) that $<_{\alpha}$ is transitive for each $\alpha$ and by Axiom $(v)$, that $<_{\beta} \subseteq<_{\alpha}$ for $\alpha \leq \beta$. In [4] it is shown that assuming irreflexivity for $<_{\alpha}$, the orderings $<_{\alpha}$ define a well-order order on $\mathbb{W}_{\alpha}$ modulo provable equivalence, provided $\Lambda$ is itself well-ordered. Thus in this case, given irreflexivity, the elements of $\mathbb{W}_{\alpha}$ can be associated with ordinals.

The next lemma is the basis of a large portion of our reasoning and we shall use it in the remainder of this paper without explicit mention.

\section{Lemma 4.3.}

1. For closed formulas $\phi$ and $\psi$, if $\beta<\alpha$, then $\mathrm{GLP}_{\Lambda} \vdash(\langle\alpha\rangle \phi \wedge\langle\beta\rangle \psi) \leftrightarrow\langle\alpha\rangle(\phi \wedge\langle\beta\rangle \psi) ;$

2. For closed formulas $\phi$ and $\psi$, if $\beta<\alpha$, then $\mathrm{GLP}_{\Lambda} \vdash(\langle\alpha\rangle \varphi \wedge[\beta] \psi) \leftrightarrow\langle\alpha\rangle(\varphi \wedge[\beta] \psi)$

3. $\mathrm{GLP}_{\Lambda} \vdash A B \rightarrow A$

4. If $A \in \mathbb{W}_{\alpha+1}$, then $\mathrm{GLP}_{\Lambda} \vdash A \wedge\langle\alpha\rangle B \leftrightarrow A \alpha B$;

5. If $A, B \in \mathbb{W}_{\alpha}$ and $\mathrm{GLP}_{\Lambda} \vdash A \leftrightarrow B$, then $\mathrm{GLP}_{\Lambda} \vdash A \alpha C \leftrightarrow B \alpha C$.

Proof. For 1, we observe that by Axiom (iv) we have $\langle\beta\rangle \psi \rightarrow[\alpha]\langle\beta\rangle \psi$, whence $\langle\alpha\rangle \phi \wedge\langle\beta\rangle \psi \rightarrow\langle\alpha\rangle(\phi \wedge\langle\beta\rangle \psi)$. For the other direction, we note that $\langle\alpha\rangle(\phi \wedge\langle\beta\rangle \psi) \rightarrow\langle\alpha\rangle\langle\beta\rangle \psi$ and the antecedent implies $\langle\beta\rangle \psi$ by Axiom (iii).

The proof of 2 is similar. By Axiom (iii) we see that $[\beta] \psi \rightarrow[\alpha][\beta] \psi$, whence $\langle\alpha\rangle \phi \wedge[\beta] \psi \rightarrow\langle\alpha\rangle(\phi \wedge[\beta] \psi)$. For the other direction, we use Axiom (iv) to get $\langle\beta\rangle \neg \psi \rightarrow[\alpha]\langle\beta\rangle \neg \psi$. Thus,

$$
\begin{aligned}
\langle\alpha\rangle(\phi \wedge[\beta] \psi) \wedge\langle\beta\rangle \neg \psi & \rightarrow\langle\alpha\rangle \perp \\
& \rightarrow \perp,
\end{aligned}
$$


whence $\langle\alpha\rangle(\phi \wedge[\beta] \psi) \rightarrow[\beta] \psi$.

Item 3 is proven by induction on the length of $A$. For zero length we see that $A=\top$. For the inductive case we reason in $\operatorname{GLP}_{\Lambda}$ and consider $\langle\alpha\rangle A B$. By a necessitation on the induction hypothesis we get $[\alpha](A B \rightarrow A)$. Using Axiom $(i)$, we see that $\langle\alpha\rangle A B \wedge[\alpha](A B \rightarrow A) \rightarrow\langle\alpha\rangle A$. We shall later see that in general $\nvdash A B \rightarrow B$.

Item 4 follows from repeatedly applying 1 (from outside in), and Item 5 follows from Item 4 .

Using the $<_{\alpha}$ relation we can define a normal form for worms.

Definition 4.4 (worm normal form). A worm $A \in \mathbb{W}$ is in WNF (worm normal form) iff

1. $A=\epsilon$, or

2. $A$ is of the form $A_{k} \alpha \ldots \alpha A_{1}$ with $\alpha=\min (A), k \geq 1$ and $A_{i} \in \mathbb{W}_{\alpha+1}$ such that each $A_{i}$ is in WNF and moreover $A_{i+1} \leq_{\alpha+1} A_{i}$ for each $i<k$.

We note that the definition of WNF refers to provability in $\mathrm{GLP}_{\Lambda}$ every time it states $A_{i+1} \leq_{\alpha+1} A_{i}$ : recall that the latter is short for $\mathrm{GLP}_{\Lambda} \vdash A_{i} \rightarrow$ $\langle\alpha+1\rangle A_{i+1}$ or $\mathrm{GLP}_{\Lambda} \vdash A_{i} \leftrightarrow A_{i+1}$. In virtue of Theorem 3.2 we can replace the use of $\mathrm{GLP}_{\Lambda}$ by its relevant fragment of finite signature.

Lemma 4.5. Each worm of width one is in WNF.

Proof. This is immediate if we conceive $\alpha^{n}$ as $\epsilon \alpha \epsilon \ldots \epsilon \alpha \epsilon$.

We emphasize that WNFs on worms are rather similar in form to Cantor normal forms (CNF) with base $\omega$ on ordinals. A notable difference is that where ordinals in CNF have their largest terms on the left-hand side, worms have their largest "term" on the right-hand side.

Lemma 4.6 below tells us that, in order to compare two worms in WNF it suffices to compare, just as with CNFs, the largest non-equal components. As a slight abuse of notation, we will often write a worm $A$ in the form $A_{k} \alpha \ldots A_{1}$, with the understanding that $A=\epsilon$ when $k=0$ and $A=A_{1}$ when $k=1$.

Lemma 4.6. Let $A=A_{k} \alpha \ldots A_{1} \alpha A^{\prime}$ be in WNF with $\alpha=\min (A)$, and each $A_{i} \in \mathbb{W}_{\alpha+1}$. Moreover, let $B$ be in WNF. We have that

$$
\text { if } A^{\prime}<_{\alpha+1} B \text {, then } A<_{\alpha+1} B \text {. }
$$


Proof. By induction on $k$. We write $A$ as $A_{k} \alpha C$. As $A_{k} \alpha C$ is in WNF and $\alpha=\min (A)$, we see that necessarily $C$ is of the form $D \alpha E$ with $D \in \mathbb{W}_{\alpha+1}$ and the $\alpha E$ part possibly empty. By the IH (or by assumption in case $k=0$ ), we see that $C<_{\alpha+1} B$, from which we obtain

$$
\begin{aligned}
B & \rightarrow\langle\alpha+1\rangle C \\
& \rightarrow\langle\alpha+1\rangle(D \alpha E) \\
& \rightarrow\langle\alpha+1\rangle D \wedge\langle\alpha+1\rangle C \quad \text { as } D \geq_{\alpha+1} A_{k} \\
& \rightarrow\langle\alpha+1\rangle A_{k} \wedge\langle\alpha+1\rangle C \\
& \rightarrow\langle\alpha+1\rangle A_{k} \wedge\langle\alpha\rangle C \\
& \rightarrow\langle\alpha+1\rangle A_{k} \alpha C .
\end{aligned}
$$

In other words, $A_{k} \alpha C<_{\alpha+1} B$ and we are done. Note that the proof also works for $A^{\prime}=\epsilon$ in which case $A$ is just of the form $\alpha^{m}$ for some $m \in \omega$.

Let us introduce some special notation for worms in WNF.

Definition 4.7. We denote $\mathbb{W}_{\alpha} \cap W N F$ by $\mathbb{W}_{\alpha}^{\circ}$.

Lemma 4.8. For all $A, B \in \mathbb{W}_{\alpha}^{\circ}$, either $A=B, A<_{\alpha} B$ or $B<_{\alpha} A$.

Proof. We may assume that $\alpha \in A B$. For if this were not the case, we prove the lemma for $A, B \in \mathbb{W}_{\beta}$ where $\beta=\min (A, B)$ and see that $\mathrm{GLP}_{\Lambda} \vdash$ $A \rightarrow\langle\beta\rangle B$ implies $\mathrm{GLP}_{\Lambda} \vdash A \rightarrow\langle\alpha\rangle B$. In case $\beta$ does not exist we have $A B=A=B=\epsilon$.

We will prove the lemma by induction on $w(A B)$. Recall that by our convention, $A_{k} \alpha \ldots A_{1} \alpha A^{\prime}$ should be understood to denote $A^{\prime}$ for $k=0$ and $A_{1} \alpha A^{\prime}$ for $k=1$.

For $w(A B) \leq 1$ and $A \neq B$ we see that $l(A)<l(B) \Rightarrow A<_{\alpha} B$ thus obtaining our result as either $l(A)<l(B)$ or $l(B)<l(A)$.

We now consider $w(A B)>1$. Suppose that $A \neq B$. We may assume that none of $A$ or $B$ is a proper extension of the other, for if, for example, $B$ were a proper extension of $A$, then $A<_{\alpha} B$ by Axiom (iii). Thus, we write $A=A_{k} \alpha \ldots A_{n} \alpha \ldots A_{1}$ and $B=B_{m} \alpha \ldots B_{n} \alpha \ldots B_{1}$ where $n$ is the smallest number such that $A_{n} \neq B_{n}$. By the IH we may, w.l.o.g. assume that $A_{n}<_{\alpha+1} B_{n}$. Reasoning in $\mathrm{GLP}_{\Lambda}$ we see that

$$
\begin{aligned}
B_{n} \rightarrow\langle\alpha+1\rangle A_{n} & \Rightarrow B_{n} \wedge \alpha \ldots B_{1} \rightarrow\langle\alpha+1\rangle A_{n} \wedge \alpha \ldots A_{1} \\
& \Rightarrow B_{n} \alpha \ldots B_{1} \rightarrow\langle\alpha+1\rangle\left(A_{n} \alpha \ldots A_{1}\right) .
\end{aligned}
$$

By Lemma 4.6 we conclude that $A<_{\alpha+1} B_{n} \alpha \ldots B_{1}$. As clearly $B_{n} \alpha \ldots B_{1} \leq_{\alpha}$ $B$ we obtain $A<_{\alpha} B$ as desired. 
Note that it is necessary to require that $A, B \in \mathbb{W}_{\alpha}$ in the above lemma: as we shall see, the normal forms 1 and 01 are $<_{1}$-incomparable. It is easy to see that the proof of the lemma automatically yields the following corollary.

Corollary 4.9. Consider two worms $A=A_{m} \alpha \ldots \alpha A_{1}$ and $B=B_{n} \alpha \ldots \alpha B_{1}$ both in $\mathbb{W}_{\alpha}^{\circ}$ with $A_{i}, B_{j} \in \mathbb{W}_{\alpha+1}^{\circ}$, and not all the $A_{i}$ nor all the $B_{j}$ empty. Let $<_{\alpha+1}^{L}$ denote the lexicographical ordering on finite strings over $\mathbb{W}_{\alpha+1}$ induced by $<_{\alpha+1}$. We have that

$$
A<_{\alpha} B \Leftrightarrow\left(A_{1}, \ldots, A_{m}\right)<_{\alpha+1}^{L}\left(B_{1}, \ldots, B_{n}\right) .
$$

The above considerations are sufficient to give an effective procedure for deciding the ordering on worms, provided we have a procedure for ordering $\Lambda$.

Definition 4.10. We call a procedure $\Lambda$-effective if it is effective using an oracle for deciding $\alpha<\beta$ for $\alpha, \beta \in|\Lambda|$.

Corollary 4.11. There is a $\Lambda$-effective procedure that compares two worms in $\mathbb{W}_{\alpha}^{\circ}$.

Proof. The $\Lambda$-effective decision procedure is already present in the proof. For $w(A B) \leq 1$ deciding whether $A<_{\alpha} B$ amounts to counting and comparing the number of symbols in $A$ and $B$. For $w(A B)>1$ this amounts to checking for first checking equality. This we can do, as we can pose oracle queries on elements in the $\langle|\Lambda|,<\rangle$ ordering. If $A \neq B$, we look at the first (from the right) non-equal term in $A$ and $B$ and recursively call upon our decision procedure. Note that in this case $l(A B)$ will diminish so we have an effective bound on the amount of calls on the decision procedure.

Next we formulate an obvious corollary to lemma 4.8 that will be very useful later on.

Corollary 4.12. For each $A, B \in \mathbb{W}_{\alpha}^{\circ}$, either $\mathrm{GLP}_{\Lambda} \vdash \alpha A \rightarrow \alpha B$, or $\mathrm{GLP}_{\Lambda} \vdash$ $\alpha B \rightarrow \alpha A$.

Proof. All implications in this proof refer to implications inside $\mathrm{GLP}_{\Lambda}$. By Lemma 4.8 we have $A \leq_{\alpha} B$ or $B<_{\alpha} A$. If $A=B$ the implication is clearly provable. If $A<_{\alpha} B$, then $B \rightarrow \alpha A$ whence $\alpha B \rightarrow \alpha \alpha A$, and $\alpha B \rightarrow \alpha A$. Likewise, $B<_{\alpha} A$ implies $\alpha B \rightarrow \alpha A$.

Corollary 4.13. Given worms $A, B \in \mathbb{W}_{\alpha}^{\circ}$, there is a worm $C \in \mathbb{W}_{\alpha}$ with $\mathrm{GLP}_{\Lambda} \vdash A \wedge B \leftrightarrow C$. Moreover, we have that $\bmod (C) \subseteq \bmod (A B)$, and $l(C) \leq l(A B)$. 
Proof. By induction on $w(A B)$. The base case is trivial. For the inductive case, we assume w.l.o.g. that $\alpha \in A B$ and write $A=A_{1} \alpha A_{2}$ and $B=B_{1} \alpha B_{2}$ with at most one of $\alpha A_{2}, \alpha B_{2}$ empty and $A_{1}, B_{1} \in \mathbb{W}_{\alpha+1}^{\circ}$. We reason in $\mathrm{GLP}_{\Lambda}$. By the IH, we find some $C_{1} \leftrightarrow A_{1} \wedge B_{1}$. By Corollary 4.12 we may assume that $\alpha A_{2} \rightarrow \alpha B_{2}$. Thus, we conclude the proof by

$$
\begin{aligned}
A \wedge B & \leftrightarrow A_{1} \alpha A_{2} \wedge B_{1} \alpha B_{2} \\
& \leftrightarrow A_{1} \wedge \alpha A_{2} \wedge B_{1} \wedge \alpha B_{2} \\
& \leftrightarrow A_{1} \wedge B_{1} \wedge \alpha A_{2} \wedge \alpha B_{2} \\
& \leftrightarrow C_{1} \wedge \alpha A_{2} \wedge \alpha B_{2} \\
& \leftrightarrow C_{1} \wedge \alpha A_{2} \\
& \leftrightarrow C_{1} \alpha A_{2}
\end{aligned}
$$

Corollary 4.14. There is a $\Lambda$-effective procedure which, given two worms $A$ and $B$ in WNF, computes a worm $C$ so that $\mathrm{GLP}_{\Lambda} \vdash A \wedge B \leftrightarrow C$ with $\bmod (C) \subseteq \bmod (A B)$, and $l(C) \leq l(A B)$.

Proof. The proof of Lemma 4.13 contains a decision procedure. For $w(A B) \leq$ 1 computing the conjunction just amounts to taking the longer of $A$ or $B$.

For $w(A B)>1$ we compute $C$ as dictated by the proof of Lemma 4.13 where we use Corollary 4.11 to decide which of $\alpha A_{2} \rightarrow \alpha B_{2}$ or $\alpha B_{2} \rightarrow \alpha A_{2}$ is the case.

In Lemma 4.8 we have proved that $<_{\alpha}$ defines a linear order on the set of normal forms of $\mathbb{W}_{\alpha}$. We shall next see through a series of lemmata that each worm $A$ is equivalent in $\mathrm{GLP}_{\Lambda}$ to one in WNF. Thus, we can drop the condition of worms being in WNF in various lemmata above 4.8, 4.12, and 4.13).

Lemma 4.15. For non-empty $A \in \mathbb{W}_{\alpha+1}$ we have for any $B \in \mathbb{W}$ that

$$
\mathrm{GLP}_{\Lambda} \vdash A \alpha B \leftrightarrow A \alpha^{n} B \quad \text { for } n \in \omega \backslash\{0\} .
$$

Proof. By an easy induction on $n$.

Lemma 4.16. Let $A:=A_{1} \alpha A_{0} B$ with $\left(B=\epsilon\right.$ or $\left.B=\alpha A^{\prime}\right)$ and each of $A_{1}, A_{0}$ in $\mathbb{W}_{\alpha+1}$.

$$
\text { If } \mathrm{GLP}_{\Lambda} \vdash A_{1} \rightarrow\langle\alpha+1\rangle A_{0} \text {, then } \mathrm{GLP}_{\Lambda} \vdash A \leftrightarrow A_{1} B \text {. }
$$


Proof. We assume $\mathrm{GLP}_{\Lambda} \vdash A_{1} \rightarrow\langle\alpha+1\rangle A_{0}$. (The first direction actually holds without the assumption.)

From $A_{1} \alpha A_{0} B$ we get $A_{1} \wedge A_{0} B$. If $B$ is of the form $\alpha A^{\prime}$, from $\alpha A_{0} B$ we get $B$ by repeatedly applying Axiom (iii) from inside out. When $B=\epsilon$, we have $B$ straight away of course. Thus,

$$
\begin{aligned}
\mathrm{GLP}_{\Lambda} \vdash A & \rightarrow A_{1} \wedge \alpha A_{0} B \\
& \rightarrow A_{1} \wedge B \\
& \rightarrow A_{1} B .
\end{aligned}
$$

For the other direction we reason in $\mathrm{GLP}_{\Lambda}$ and use our assumption that $A_{1} \rightarrow\langle\alpha+1\rangle A_{0}$.

$$
\begin{aligned}
A_{1} B & \rightarrow A_{1} B \wedge\langle\alpha+1\rangle A_{0} \\
& \rightarrow A_{1} \wedge B \wedge\langle\alpha+1\rangle A_{0} \\
& \rightarrow A_{1} \wedge\langle\alpha+1\rangle A_{0} B \\
& \rightarrow A_{1} \wedge\langle\alpha\rangle A_{0} B \\
& \rightarrow A_{1} \alpha A_{0} B .
\end{aligned}
$$

Lemma 4.17. Each worm $A \in \mathbb{W}$ is equivalent in $\operatorname{GLP}_{\Lambda}$ to some $\operatorname{NF}(A)$ in $W N F$. Moreover, $\bmod (\operatorname{NF}(A)) \subseteq \bmod (A)$.

Proof. By induction on $l(A)$ we shall prove that each $A \in \mathbb{W}_{\alpha}$ is equivalent to some $C \in \mathbb{W}_{\alpha}$ with $l(C) \leq l(A)$ and $\bmod (C) \subseteq \bmod (A)$. For $l(A)=0$ we see that $A=\epsilon \in \mathrm{WNF}$. We proceed to prove the case when $l(A)>0$. All modal reasoning takes place in $\mathrm{GLP}_{\Lambda}$.

For $\alpha=\min (A)$, we use Lemma 4.15 to write $A$ as $A_{k+1} \alpha A_{k} \alpha \ldots A_{0}$ with $k \geq 0$ and each $A_{i} \in \mathbb{W}_{\alpha+1}$. Recall that $A_{0} \alpha \ldots A_{0}$ just means $A_{0}$. By the IH we find some $A_{i}^{\prime} \in \mathbb{W}_{\alpha+1}$ such that $A_{l}^{\prime} \alpha \ldots A_{0}^{\prime}$ is in WNF and equivalent to $A_{k} \alpha \ldots A_{0}$. Moreover, we have that $l\left(A_{l}^{\prime} \alpha \ldots A_{0}^{\prime}\right) \leq l\left(A_{k} \alpha \ldots A_{0}\right)$. It is easy to see that we also have that $\alpha A_{l}^{\prime} \alpha \ldots A_{0}^{\prime}$ is equivalent to $\alpha A_{k} \alpha \ldots A_{0}$.

Again, by the IH, we can find some $D \in \mathbb{W}_{\alpha+1}^{\circ}$ which is equivalent to $A_{k+1}$ and with $l(D) \leq l\left(A_{k+1}\right)$. Clearly we have that

$$
\begin{aligned}
D \alpha A_{l}^{\prime} \alpha \ldots A_{0}^{\prime} & \leftrightarrow D \wedge \alpha A_{l}^{\prime} \alpha \ldots A_{0}^{\prime} \\
& \leftrightarrow A_{k+1} \wedge \alpha A_{l}^{\prime} \alpha \ldots A_{0}^{\prime} \\
& \leftrightarrow A_{k+1} \wedge \alpha A_{k} \alpha \ldots A_{0} \\
& \leftrightarrow A .
\end{aligned}
$$

If $A_{l}^{\prime} \geq_{\alpha+1} D$ then $D \alpha A_{l}^{\prime} \alpha \ldots A_{0}^{\prime}$ is in WNF. Moreover, $l\left(D \alpha A_{l}^{\prime} \alpha \ldots A_{0}^{\prime}\right) \leq$ $l(D)+l\left(\alpha A_{l}^{\prime} \alpha \ldots A_{0}^{\prime}\right) \leq l\left(A_{k+1}\right)+l\left(\alpha A_{k} \alpha \ldots A_{0}\right) \leq l(A)$ and $D \alpha A_{l}^{\prime} \alpha \ldots A_{0}^{\prime} \in$ $\mathbb{W}_{\alpha}$. 
If $A_{l}^{\prime} \nsupseteq_{\alpha+1} D$ we conclude by Lemma 4.8 that $D>_{\alpha+1} A_{l}^{\prime}$. Now we can apply Lemma 4.16 to see that

$$
\begin{aligned}
A & \leftrightarrow D \alpha A_{l}^{\prime} \alpha \ldots A_{0}^{\prime} \\
& \leftrightarrow D \alpha A_{l}^{\prime} B \\
& \leftrightarrow D B .
\end{aligned}
$$

We conclude by yet another call upon the IH to find a WNF in $\mathbb{W}_{\alpha}$ equivalent to $D B$ and of length at most $l(D B)$.

Thus, tranforming a worm into an equivalent one in WNF boils down to repeatedly shortening the original worm by applying lemmata 4.15 and 4.16 whence it is clear that $\bmod (\operatorname{NF}(A)) \subseteq \bmod (A)$.

Corollary 4.18. Given some $\mathrm{GLP}_{\Lambda}$ worm $A \in \mathbb{W}_{\alpha}$, there is a $\Lambda$-computable procedure to obtain a worm $A^{\prime} \in \mathbb{W}_{\alpha}^{\circ}$ with $\bmod \left(A^{\prime}\right) \subseteq \bmod (A)$ and $\mathrm{GLP}_{\Lambda} \vdash$ $A \leftrightarrow A^{\prime}$.

Proof. We see that the proof of Lemma 4.17 actually contains a description of this decision procedure. In the inductive step, whether or not we have to apply Lemma 4.16 can be $\Lambda$-decided in virtue of Corollary 4.11

Now that we have seen that we can $\Lambda$-effectively compute a WNF, we conclude from Corollary 4.14 that we can $\Lambda$-compute the conjunction of any two worms $A$ and $B$. In other words, we can omit the restriction that $A$ and $B$ be in WNF in Corollary 4.14.

\section{A normal form theorem for closed formulas}

So far in this paper, no irreflexivity of the relations $<_{\alpha}$ has been used in our reasoning. In this section we shall prove that each closed formula is actually equivalent in $\mathrm{GLP}_{\Lambda}$ to a Boolean combination of worms and some important corollaries thereof. In the proofs, irreflexivity plays an essential role.

\section{$5.1 \quad$ Irreflexivity}

By irreflexivity we mean the claim that for no $A \in \mathbb{W}$ and for no $\alpha \in|\Lambda|$ do we have $\mathrm{GLP}_{\Lambda} \nvdash A \rightarrow\langle\alpha\rangle A$. In view of the following result, this is equivalent to demanding that worms be consistent.

Lemma 5.1. If $\mathrm{GLP}_{\Lambda} \vdash A \rightarrow\langle\alpha\rangle A$, then $\mathrm{GLP}_{\Lambda} \vdash \neg A$. 
Proof. If we assume $\mathrm{GLP}_{\Lambda} \vdash A \rightarrow\langle\alpha\rangle A$, then we would get by contraposition and necessitation that $\mathrm{GLP}_{\Lambda} \vdash[\alpha]([\alpha] \neg A \rightarrow \neg A)$. One application of Löb's axiom would yield $\vdash[\alpha] \neg A$. Using the contraposition of our assumption again, we obtain $\mathrm{GLP}_{\Lambda} \vdash \neg A$.

Fortunately, irreflexivity does hold. This is known for well-ordered $\Lambda$, in which case there are many arguments in the literature as to why that is, each with its advantages and disadvantages.

Arithmetic interpretations. In case of $\mathrm{GLP}_{\omega}$ all formulas $\psi$ come with a clearly defined arithmetical interpretation $\psi^{\star}$ where each $[n]$ is interpreted as a natural formalization of "provable in EA together all true $\Pi_{n}$-sentences" 20]. The soundness for this interpretation tells us that for any formula $\varphi$ and any interpretation $\star$ mapping propositional variables to sentences in the language of arithmetic we have that $\mathrm{GLP}_{\omega} \vdash \varphi \Rightarrow \mathrm{PA} \vdash \varphi^{\star}$. In particular we get for worms $A$ that $\mathrm{GLP}_{\omega} \vdash \neg A \Rightarrow \mathrm{PA} \vdash \neg A^{\star}$. Now $\neg A^{\star}$ is just an iteration of inconsistency assertions all of which are not provable by PA as everything provable by PA is actually true. This reasoning, although using quite some heavy machinery as reflection over PA, establishes the irreflexivity of $<_{n}$ in $\mathrm{GLP}_{\omega}$. Recent work by the authors and Dashkov suggests that this may be generalized to larger recursive ordinals than $\omega$, however arithmetic interpretations for non-recursive ordinals or for linear orders that are not well-founded are not currently known.

Kripke semantics. Kripke semantics for $\mathrm{GLP}_{\omega}$ have been studied extensively [20, 22, 2, 18]. Using these semantics it is easy to see that for each $n \in \omega$, and each worm $A \in \mathrm{GLP}_{\omega}$ we can find a model $\mathcal{M}$ and a world $x$ of $\mathcal{M}$ where both $A$ and $[n] \neg A$ hold, thus establishing the irreflexivity of $<_{n}$ in $\mathrm{GLP}_{\omega}$. More recently this has been extended to $\mathrm{GLP}_{\Lambda}$ for an arbitrary ordinal $\Lambda$ [15]. One drawback is that the methods used are not strictly finitary, whereas [2] gives a full finitary treatment of $\mathrm{GLP}_{\omega}^{0}$. Thus the irreflexivity of $\mathrm{GLP}_{\omega}$ can be proven on strictly finitary grounds. As before, the assumption that $\Lambda$ is well-ordered plays an important role and it is not obvious how one could generalize these methods, however they do have the advantage of working for arbitrary ordinals, including uncountable ones.

Topological semantics. The same reasoning can also be performed using topological semantics of GLP $\omega$ [19, 18, 1, which likewise have been generalized to arbitrary ordinals in [13. As before, however, the methods used in the transfinite setting are not strictly finitary and have been developed only 
for well-ordered $\Lambda$.

Now that we have provided a reduction from $\operatorname{GLP}_{\Lambda}$ to $\mathrm{GLP}_{\omega}$ in Theorem 3.2, we in particular have a reduction from $\mathrm{GLP}_{\Lambda}^{0}$ to $\mathrm{GLP}_{\omega}^{0}$. This gives us a new proof of irreflexivity for the general logic. The present argument is both the first finitary proof of irreflexivity for infinite orders different from $\omega$, provided that $\Lambda$ (and hence $G_{L} P_{\Lambda}$ ) can be represented in a finitary framework such as Primitive Recursive Arithmetic, as well as the first proof of irreflexivity which does not require that $\Lambda$ be well-founded.

Theorem 5.2. For each linear order $\Lambda$ and each $\alpha \in|\Lambda|$, the relation $<_{\alpha}$ is irreflexive on $\mathbb{W}$.

Proof. The relation $<_{n}$ is known to be irreflexive over $\mathrm{GLP}_{\omega}$, and this fact may be proven by finitary means [22, 2]. Moreover, if for some worm $A$ we had that $\operatorname{GLP}_{\Lambda} \vdash(A \rightarrow\langle\alpha\rangle A)=\psi$, then we would have that $\mathrm{GLP}_{\omega} \vdash \hat{\psi}$, contradicting the irreflexivity of $<_{n}$ for some $n$.

Thus, we have shown that $<_{\alpha}$ is transitive and irreflexive and defines a linear order on the worm normal forms in $\mathbb{W}_{\alpha}$. In fact, in [4] it has been shown to be a well-order on $\mathbb{W}_{\alpha}$, if it is irreflexive and $\Lambda$ is well-founded. In particular, if we allow $\Lambda$ to be the clas of all ordinals, there is a one-one correspondence between normal forms in $\mathbb{W}$ and ordinals in On. In [17] the relation $<_{\alpha}$ is also studied and seen to be a non-tree-like partial well-order on $\mathbb{W}$.

Without using irreflexivity we proved two major results on worms and WNFs. First, that WNFs are linearly ordered by $<_{0}$, and second, that each worm is equivalent to one in WNF. Using irreflexivity we readily see that the WNFs actually form a strict linear order under $<_{0}$ and that each formula is equivalent to a unique WNF.

Lemma 5.3. Each worm $A$ is equivalent in $\operatorname{GLP}_{\Lambda}$ to a unique worm $\operatorname{NF}(A)$ in WNF.

Proof. Suppose for a contradiction that $A$ had over GLP ${ }_{\Lambda}$ two different WNFs $B$ and $C$. Then, by Lemma 4.8 and reasoning in $\mathrm{GLP}_{\Lambda}$ we may assume that $B \rightarrow\langle\alpha\rangle C$ where $\alpha=\min (A)$. Thus,

$$
\begin{aligned}
A & \rightarrow B \\
& \rightarrow\langle\alpha\rangle C \\
& \rightarrow\langle\alpha\rangle A,
\end{aligned}
$$


which contradicts irreflexivity.

Using irreflexivity it also immediate that our new definition of normal forms is equivalent to the one previously used in the literature. In the remainder of this paper we shall freely use irreflexivity.

\subsection{Closed formulas and worms}

In this section we shall show that each closed formula is equivalent to a Boolean combination of worms. We follow Section 3 of [4] very closely, formulating slightly stronger versions of the lemmata in [4] leading up to important further observations.

The first lemma of this section in a sense tells us that whatever piece of genuine information we add to a worm, this will always increase the consistency strength of it (equivalently, increase the corresponding ordertype).

Lemma 5.4. Let $A, A_{1}, \ldots A_{I} \in \mathbb{W}_{\alpha}$ be such that for each $i \leq I, \mathrm{GLP}_{\Lambda} \nvdash$ $A \rightarrow A_{i}$. Then it follows that $\mathrm{GLP}_{\Lambda} \vdash A \wedge \bigvee_{i=1}^{I} A_{i} \rightarrow\langle\alpha\rangle A$.

Proof. All modal reasoning will be in $\mathrm{GLP}_{\Lambda}$. By Corollary 4.13 for each $i$, let $\operatorname{Conj}\left(A, A_{i}\right)$ be the worm in $\mathbb{W}_{\alpha}^{\circ}$ that is equivalent to $A \wedge A_{i}$. By Lemma 4.8 we can $<_{\alpha}$-compare $\operatorname{Conj}\left(A, A_{i}\right)$ to $A$. However, $\operatorname{Conj}\left(A, A_{i}\right)=A$ contradicts $\nvdash A \rightarrow A_{i}$. Likewise, $\operatorname{Conj}\left(A, A_{i}\right)<_{\alpha} A$ contradicts the irreflexivity of $<_{\alpha}$. We conclude that $\operatorname{Conj}\left(A, A_{i}\right) \rightarrow\langle\alpha\rangle A$ whence $A \wedge A_{i} \rightarrow\langle\alpha\rangle A$. As $i$ was arbitrary, we obtain $A \wedge \bigvee_{i=1}^{I} A_{i} \rightarrow\langle\alpha\rangle A$.

A direct and nice corollary to this lemma is that worms satisfy a certain form of disjunction property.

Corollary 5.5. For $A, A_{i} \in \mathbb{W}$ we have that

$$
\mathrm{GLP}_{\Lambda} \vdash A \rightarrow \bigvee_{i=1}^{I} A_{i} \quad \Leftrightarrow \quad \text { for some } i \leq I, \mathrm{GLP}_{\Lambda} \vdash A \rightarrow A_{i}
$$

Proof. We reason about derivability in $\mathrm{GLP}_{\Lambda}$ by contraposition and suppose that for each $i \leq I, \nvdash A \rightarrow A_{i}$. Then, by Lemma 5.4 we obtain that $\vdash A \wedge \bigvee_{i=1}^{I} A_{i} \rightarrow\langle 0\rangle A$. Irreflexivity of $<_{0}$ imposes that $\nvdash A \rightarrow \bigvee_{i=1}^{I} A_{i}$, as required.

Lemma 5.6. For $A, A_{1}, \ldots A_{k} \in \mathbb{W}_{\alpha}$ we have in $\mathrm{GLP}_{\Lambda}$ that either

- $\langle\alpha\rangle\left(A \wedge \bigwedge_{i} \neg A_{i}\right) \leftrightarrow\langle\alpha\rangle$, or that 
- $A \wedge \bigwedge_{i} \neg A_{i} \leftrightarrow \perp$ whence also $\langle\alpha\rangle\left(A \wedge \bigwedge_{i} \neg A_{i}\right) \leftrightarrow \perp$.

Proof. All modal reasoning will concern $\mathrm{GLP}_{\Lambda}$. In case that for some $i$ we have that $\vdash A \rightarrow A_{i}$, clearly $\langle\alpha\rangle\left(A \wedge \bigwedge_{i} \neg A_{i}\right) \leftrightarrow \perp$. In case that for no $i$, $\vdash A \rightarrow A_{i}$ we apply Lemma 5.4 .

$$
\begin{array}{rlrl}
{[\alpha]\left(A \rightarrow \bigvee_{i} A_{i}\right)} & \rightarrow[\alpha]\left(A \rightarrow\left(A \wedge \bigvee_{i} A_{i}\right)\right) & & \text { by Lemma } 5.4 \\
& \rightarrow[\alpha](A \rightarrow\langle\alpha\rangle A) & & \text { by Löb's axiom } \\
& \rightarrow[\alpha] \neg A & \\
& \rightarrow[\alpha]\left(A \rightarrow \bigvee_{i} A_{i}\right) &
\end{array}
$$

Thus, $[\alpha]\left(A \rightarrow \bigvee_{i} A_{i}\right) \leftrightarrow[\alpha] \neg A$, whence $\langle\alpha\rangle\left(A \wedge \bigwedge_{i} \neg A_{i}\right) \leftrightarrow\langle\alpha\rangle A$.

Corollary 5.7. For any worm $A \in \mathbb{W}$, and $A_{1}, \ldots A_{k} \in \mathbb{W}_{\alpha}$ we have in $\mathrm{GLP}_{\Lambda}$ that either

- $\langle\alpha\rangle\left(A \wedge \bigwedge_{i} \neg A_{i}\right) \leftrightarrow\langle\alpha\rangle A$, or that

- $A \wedge \bigwedge_{i} \neg A_{i} \leftrightarrow \perp$ whence also $\langle\alpha\rangle\left(A \wedge \bigwedge_{i} \neg A_{i}\right) \leftrightarrow \perp$.

Proof. We can split $A$ into the largest prefix $A_{\alpha}$ of $A$ that belongs to $\mathbb{W}_{\alpha}$ and the remainder $A_{<\alpha}$ of $A$. Consequently, $A_{<\alpha}$ starts with a symbol smaller than $\alpha$ or is empty and we have $A=A_{\alpha} A_{<\alpha} \leftrightarrow A_{\alpha} \wedge A_{<\alpha}$. Thus,

$$
\begin{aligned}
\langle\alpha\rangle\left(A \wedge \bigwedge_{i} \neg A_{i}\right) & \leftrightarrow\langle\alpha\rangle\left(A_{\alpha} \wedge A_{<\alpha} \wedge \bigwedge_{i} \neg A_{i}\right) \\
& \leftrightarrow A_{<\alpha} \wedge\langle\alpha\rangle\left(A_{\alpha} \wedge \bigwedge_{i} \neg A_{i}\right) \quad \text { first case of Lemma } 5.6 \\
& \leftrightarrow A_{<\alpha} \wedge\langle\alpha\rangle A_{\alpha} \\
& \leftrightarrow\langle\alpha\rangle\left(A_{\alpha} \wedge A_{<\alpha}\right) \\
& \leftrightarrow\langle\alpha\rangle A .
\end{aligned}
$$

Note that in the second case of Lemma 5.6 we end up with $\perp$ as desired.

Lemma 5.8. Let $\phi\left(A_{1}, \ldots, A_{n}\right)$ be a Boolean combination of the worms $A_{1}, \ldots, A_{n}$. Then $\langle\alpha\rangle \phi\left(A_{1}, \ldots, A_{n}\right)$ is equivalent in $\mathrm{GLP}_{\Lambda}$ to some formula $\operatorname{Diamond}_{\alpha}(\phi)$ which is a disjunction of conjunctions of worms or negated worms such that non-empty worms that are not negated have a first modality $\alpha$ and non-empty worms that are negated have a first modality strictly less than $\alpha$. Moreover, we have that $\bmod \left(\operatorname{Diamond}_{\alpha}(\phi)\right) \subseteq\{\alpha\} \cup \bmod (\phi)$.

Proof. All modal reasoning concerns $\mathrm{GLP}_{\Lambda}$. Any word $A_{i}$ in $\phi\left(A_{1}, \ldots, A_{n}\right)$ is equivalent to some $B_{i} \wedge C_{i}$ where $B_{i} \in \mathbb{W}_{\alpha}$ and such that the first element of $C_{i}$ is less than $\alpha$. Thus, $\phi\left(A_{1}, \ldots, A_{n}\right)$ is equivalent to some other Boolean combination $\psi\left(B_{1}, \ldots, B_{n}, C_{1}, \ldots, C_{n}\right)$ of the worms $B_{1}, \ldots, B_{n}, C_{1}, \ldots, C_{n}$. 
We write $\psi$ in disjunctive normal form. In the remainder of this proof we shall not be too precise in writing indices and subindices as the context should make clear what is meant. As $\langle\alpha\rangle \bigvee_{j} \chi_{j} \leftrightarrow \bigvee_{i}\langle\alpha\rangle \chi_{j}$, it suffices to prove the lemma for formulas of the form $\bigwedge_{i} \pm D_{i}$ where each $D_{i} \in$ $\left\{B_{1}, \ldots, B_{n}, C_{1}, \ldots, C_{n}\right\}$. By Lemma 4.3 we see that

$$
\langle\alpha\rangle \bigwedge_{i} \pm D_{i} \leftrightarrow \bigwedge_{j} \pm C_{i} \wedge\langle\alpha\rangle \bigwedge_{k} \pm B_{i}
$$

As worms are closed under taking conjunctions, we can write $\bigwedge_{k} \pm B_{i}$ of the form $B \wedge \bigwedge_{l} \neg C_{l}$ where each of $B, C_{l} \in \mathbb{W}_{\alpha}$.

Now we can apply Lemma 5.6 to obtain $\langle\alpha\rangle \bigwedge_{k} \pm B_{k} \leftrightarrow\langle\alpha\rangle B$, and

$$
\langle\alpha\rangle \bigwedge_{i} \pm D_{i} \leftrightarrow \bigwedge_{j} \pm C_{j} \wedge\langle\alpha\rangle B
$$

All the positive worms in $\bigwedge_{i} \pm C_{i}$ can be moved as conjunctions under the $\langle\alpha\rangle$ modality of $\langle\alpha\rangle B$ again to form a single worm as the conjunctions of all those worms are equivalent to a single one.

Corollary 5.9. Each closed formula $\phi$ is equivalent in $\mathrm{GLP}_{\Lambda}$ to a Boolean combination $\operatorname{BCW}(\phi)$ of worms such that $\bmod (\operatorname{BCW}(\phi)) \subseteq \bmod (\phi)$.

Proof. By induction on the complexity of $\psi$. The only interesting case is $\langle\alpha\rangle$ which is taken care of by Lemma 5.8. Note that in principle $\mathrm{BCW}(\phi)$ need not be unique as, for example, one could consider various equivalent disjunctive normal forms along the way of constructing $\operatorname{BCW}(\phi)$.

Corollary 5.10. For each closed formula $\psi$ of $\mathrm{GLP}_{\Lambda}$ we can $\Lambda$-effectively compute an $\mathrm{GLP}_{\Lambda}$-equivalent formula $\chi$ which is a Boolean combination of worms such that $\bmod (\chi) \subseteq \bmod (\phi)$.

Proof. By inspection of the proofs of Lemma 5.8 and Lemma 5.6 we can retrieve a $\Lambda$-effective recipe. We use that we already know that we can $\Lambda$-effectively compare two worms and compute their conjunction.

Corollary 5.11. For each consistent closed formula $\phi$ there is a worm $A$ with $\bmod (A)=\bmod (\phi) \cup\{0\}$ so that $\mathrm{GLP}_{\Lambda} \vdash\langle 0\rangle \phi \leftrightarrow A$.

Moreover, $\mathrm{GLP}_{\Lambda}^{0} \vdash\langle\max (A)\rangle^{l(A)} \top \rightarrow A$.

Proof. Write $\phi$ in disjunctive normal form where the atoms are worms. As $\langle 0\rangle$ distributes over our disjunction, to each disjunct we apply Lemma 5.6. As $\varphi$ was consistent, so is each of the disjuncts whence each disjunct is 
equivalent $\langle 0\rangle A_{i}$ for some worm $A_{i}$. Thus, we end up with a disjunction of worms that start with a $\langle 0\rangle$ modality. Corollary 4.12 tells us that there is a 'minimal' disjunct and thus we see that such a disjunction can actually be replaced by a single disjunct.

By an easy proof similar to that of Lemma 4.15, we further see that

$$
\mathrm{GLP}_{\Lambda}^{0} \vdash\langle\max (A)\rangle^{l(A)} \top \rightarrow A,
$$

from which our second claim immediately follows.

Corollary 5.11 has an important consequence for the model theory of $\mathrm{GLP}_{\Lambda}^{0}$. This result is used in [13] to give a completeness proof for certain models of the closed fragment. Namely, if we have a Kripke frame $\mathfrak{F}$ such that $\mathfrak{F} \models G^{\prime} P_{\Lambda}^{0}$ and we wish to check that $\mathrm{GLP}_{\Lambda}^{0}$ is moreover complete for $\mathfrak{F}$, it suffices to check that $\mathfrak{F}$ satisfies enough worms:

Corollary 5.12. Suppose $\mathfrak{F}=\left\langle W,\left\langle R_{\xi}\right\rangle_{\xi<\Lambda}\right\rangle$ is any Kripke frame such that $\mathfrak{F} \models \mathrm{GLP}_{\Lambda}^{0}$ and, for all $\lambda<\Lambda$ and $n<\omega$, there is $w \in W$ such that $\mathfrak{F}, w \models\langle\lambda\rangle^{n} \top$.

Then, for every consistent closed formula $\phi$ there is $w \in W$ such that $\mathfrak{F}, w \models \phi$.

If $\Lambda$ is a limit ordinal, it suffices to consider $n=1$.

Proof. Suppose that $\mathfrak{F}, w \models\langle\lambda\rangle^{n} \top$ for all $n<\omega$ and $\lambda<\Lambda$ and $\phi$ is consistent.

Then we have in particular that for some $w \in W, \mathfrak{F}, v \models\langle\max \phi\rangle^{l(\phi)} \top$, so that by Corollary 5.11 we also have $\mathfrak{F}, v \models\langle 0\rangle \phi$. But then we have $w$ with $v R_{0} w$ and $\mathfrak{F}, w \models \phi$, i.e., $\phi$ is satisfied on $\mathfrak{F}$, as claimed.

If $\Lambda$ is a limit ordinal we observe that

$$
\mathrm{GLP}_{\Lambda}^{0} \vdash\langle\max \phi+1\rangle \top \rightarrow\langle\max \phi\rangle^{l(\phi)} \top,
$$

so we may choose $v$ satisfying $\langle\max \phi+1\rangle \top$ instead.

Note that this corollary is here stated for Kripke semantics but actually holds true for any reasonable notion of $\mathrm{GLP}_{\Lambda}$ semantics.

\section{Alternative axiomatizations}

In 2 it was observed that one could simultaneously restrict Löb's axiom and the monotonicity axiom $\langle\alpha\rangle \phi \rightarrow\langle\beta\rangle \phi$ for $\alpha \geq \beta$ to worms and still obtain a full axiomatization of $\mathrm{GLP}_{\omega}^{0}$. In this section we shall prove that we can also 
simultaneously restrict the axiom of negative introspection $\langle\alpha\rangle \phi \rightarrow[\beta]\langle\alpha\rangle \phi$ with $\alpha<\beta$ to worms and still obtain a full axiomatization of $\mathrm{GLP}_{\omega}^{0}$. In order to prove this, we need to recall the decision procedure as exposed in [4.

\subsection{A decision procedure}

Theorem 6.1. There is a $\Lambda$-effective decision procedure for $\mathrm{GLP}_{\Lambda}^{0} \vdash \phi$.

Proof. We shall first outline a decision procedure and then see that this is indeed effective. By Corollary [5.9 we know that each closed formula $\phi$ is equivalent in $\mathrm{GLP}_{\Lambda}$ to a Boolean combination of worms. We can write this Boolean combination in conjunctive normal form and as worms are closed under conjunctions, each conjunct can be written of the form $A_{i} \rightarrow \bigvee_{j} B_{i j}$ with each $A_{i}$ and $B_{i j}$ in WNF. Let us call this the worm normal form and we write $\operatorname{WNF}(\varphi)$.

The decision procedure is represented by the following scheme:

$$
\begin{array}{rlrl}
\operatorname{GLP}_{\Lambda}^{0} \vdash \phi & \Leftrightarrow & \operatorname{GLP}_{\Lambda}^{0} \vdash \operatorname{WNF}(\phi) & \\
& \Leftrightarrow \operatorname{GLP}_{\Lambda}^{0} \vdash \bigwedge_{i}\left(A_{i} \rightarrow \bigvee_{j} B_{i j}\right) & \\
& \Leftrightarrow \forall i \mathrm{GLP}_{\Lambda}^{0} \vdash A_{i} \rightarrow \bigvee_{j} B_{i j} & & \text { by Lemma } 5.5 \\
& \Leftrightarrow \forall i \exists j \mathrm{GLP}_{\Lambda}^{0} \vdash A_{i} \rightarrow B_{i j} & \\
& \Leftrightarrow \quad \forall i \exists j \mathrm{GLP}_{\Lambda}^{0} \vdash A_{i} \leftrightarrow A_{i} \wedge B_{i j} & & \text { by Lemma } 5.3 \\
& \Leftrightarrow & & \text { and Corollary } 4.13 \\
& & \bmod (\phi) \subseteq \Lambda \text { and } &
\end{array}
$$

The $\bmod (\phi) \subseteq \Lambda$ in the last line we have in virtue of our conservation result as stated in 3.2. In order to see that the above equivalences yield a $\Lambda$ effective decision procedure, there are three major things that we need to check.

1. $\operatorname{WNF}(\phi)$ can be $\Lambda$-effectively computed from a closed formula $\phi$;

2. $\mathrm{NF}(A)$ can be $\Lambda$-effectively computed from a worm $A$;

3. The worm corresponding to $A \wedge B$ can be $\Lambda$-effectively computed from $A$ and $B$.

But, Item 3 is just Corollary 4.14, Item 2 is just Corollary 4.18, and Item 1 follows directly from Corollary 5.10 and Corollary 4.14. 
In practice we will always only be interested in notation systems that are easy, say primitive recursive, for which the following corollary is relevant.

Corollary 6.2. For each effective ordinal $\Lambda$, there is an effective decision procedure for $\mathrm{GLP}_{\Lambda}^{0} \vdash \phi$.

In virtue of Theorem 3.2 we knew already that $\mathrm{GLP}_{\Lambda}^{0}$ has a very easy reduction to $\mathrm{GLP}_{\omega}^{0}$ where the latter is know tho be PSPACE complete.

Corollary 6.3. If the ordering on $\Lambda$ is decidable in poly-time, then the computational complexity of $\mathrm{GLP}_{\Lambda}^{0}$ is PSPACE complete.

Proof. Theorem 3.2, provides a poly-time reduction from $\mathrm{GLP}_{\Lambda}^{0}$ to $\mathrm{GLP}_{\omega}^{0}$. Although the closed fragment for GL is decidable in PTIME ([10]), Pakhomov has shown ([23]) that the closed fragment of GLP $\omega$ is PSPACE complete.

\subsection{Restricting to worms}

We are now ready to prove the main theorem of this section. By $w-G L P{ }_{\Lambda}^{0}$ we denote the logic that is as $\operatorname{GLP}_{\Lambda}^{0}$ but the axioms

$$
\begin{array}{ll}
{[\alpha]([\alpha] A \rightarrow A) \rightarrow[\alpha] A} & \\
\langle\alpha\rangle A \rightarrow\langle\beta\rangle A & \alpha \geq \beta \\
\langle\alpha\rangle A \rightarrow[\beta]\langle\alpha\rangle A & \alpha<\beta
\end{array}
$$

restricted to worms $A$.

Theorem 6.4. The logics $\mathrm{w}-\mathrm{GLP}_{\Lambda}^{0}$ and $\mathrm{GLP}_{\Lambda}^{0}$ prove the same set of theorems.

Proof. We will first prove

$$
\begin{aligned}
& \langle\alpha\rangle \phi \rightarrow\langle\beta\rangle \phi \quad \text { for } \alpha \geq \beta \text { and } \\
& \langle\alpha\rangle \phi \rightarrow[\beta]\langle\alpha\rangle \phi \quad \text { for } \alpha<\beta
\end{aligned}
$$

for $\phi$ any closed formula within $\mathrm{w}-\mathrm{GLP}_{\Lambda}^{0}$. We write $\phi$ in disjunctive normal form as $\bigvee_{i}\left(A_{i} \wedge \bigwedge_{j} \neg B_{i j} \wedge \bigwedge_{k} \neg C_{i k}\right)$ where each $B_{i j} \in \mathbb{W}_{\alpha}$ and each $C_{i k}$ starts with a modality smaller than $\alpha$.

When $\langle\alpha\rangle \phi \leftrightarrow \perp$ there is nothing to prove, so we may assume that $\nvdash A_{i} \rightarrow B_{i j}$ and $\nvdash A_{i} \rightarrow C_{i k}$ and use Corollary 5.7 to see that for each $i$ we have that

$$
\begin{aligned}
\langle\alpha\rangle\left(A_{i} \wedge \bigwedge_{j} \neg B_{i j} \wedge \bigwedge_{k} \neg C_{i k}\right) & \leftrightarrow\langle\alpha\rangle\left(A_{i} \wedge \bigwedge_{j} \neg B_{i j} \wedge \bigwedge_{k} \neg C_{i k}\right) \\
& \leftrightarrow \bigwedge_{k} \neg C_{i k} \wedge\langle\alpha\rangle\left(A_{i} \wedge \bigwedge_{j} \neg B_{i j}\right) \\
& \leftrightarrow \bigwedge_{k} \neg C_{i k} \wedge\langle\alpha\rangle A_{i} \\
& \leftrightarrow\langle\alpha\rangle\left(A_{i} \wedge \bigwedge_{k} \neg C_{i k}\right) .
\end{aligned}
$$


Let us first see that $\langle\alpha\rangle \phi \rightarrow\langle\beta\rangle \phi$ for $\alpha \geq \beta$. We observe that $\mathbb{W}_{\alpha} \subset \mathbb{W}_{\beta}$. We shall write $\bigwedge_{k} \neg C_{i k}$ as $\bigwedge_{k^{\prime}} \neg C_{i k^{\prime}} \wedge \bigwedge_{l} \neg D_{i l}$ where the first modality in each $C_{i k^{\prime}}$ is strictly below $\beta$ and the first modality in each $D_{i l}$ is between $\beta$ and strictly below $\alpha$.

$$
\begin{aligned}
& \langle\alpha\rangle \phi \rightarrow\langle\alpha\rangle \bigvee_{i}\left(A_{i} \wedge \bigwedge_{j} \neg B_{i j} \wedge \bigwedge_{k} \neg C_{i k}\right) \\
& \rightarrow \bigvee_{i}\langle\alpha\rangle\left(A_{i} \wedge \bigwedge_{j} \neg B_{i j} \wedge \bigwedge_{k} \neg C_{i k}\right) \\
& \rightarrow \bigvee_{i}\left(\bigwedge_{k} \neg C_{i k} \wedge\langle\alpha\rangle\left(A_{i} \wedge \bigwedge_{j} \neg B_{i j}\right)\right) \\
& \rightarrow \bigvee_{i}\left(\bigwedge_{k} \neg C_{i k} \wedge\langle\alpha\rangle A_{i}\right) \\
& \rightarrow \bigvee_{i}\left(\bigwedge_{k} \neg C_{i k} \wedge\langle\beta\rangle A_{i}\right) \\
& \rightarrow \bigvee_{i}\left(\bigwedge_{k^{\prime}} \neg C_{i k^{\prime}} \wedge \bigwedge_{l} \neg D_{i l} \wedge\langle\beta\rangle A_{i}\right) \\
& \rightarrow \bigvee_{i}\left(\bigwedge_{k^{\prime}} \neg C_{i k^{\prime}} \wedge\langle\beta\rangle A_{i}\right) \\
& \left.\rightarrow \bigvee_{i}\langle\beta\rangle\left(A_{i} \wedge \bigwedge_{k^{\prime}} \neg C_{i k^{\prime}}\right)\right) \\
& \rightarrow \bigvee_{i}\langle\beta\rangle\left(A_{i} \wedge \bigwedge_{j} \neg B_{i j} \wedge \bigwedge_{k^{\prime}} \neg C_{i k^{\prime}} \wedge \bigwedge_{l} \neg D_{i l}\right) \\
& \rightarrow\langle\beta\rangle \bigvee_{i}\left(A_{i} \wedge \bigwedge_{j} \neg B_{i j} \wedge \bigwedge_{k^{\prime}} \neg C_{i k^{\prime}} \wedge \bigwedge_{l} \neg D_{i l}\right) \\
& \rightarrow\langle\beta\rangle \phi \text {. } \\
& \text { As } \nvdash A_{i} \rightarrow D_{i l}
\end{aligned}
$$

For the proof of $\langle\alpha\rangle \phi \rightarrow[\beta]\langle\alpha\rangle \phi$ for $\alpha<\beta$ it clearly suffices to show for each $i$ that

$$
\langle\alpha\rangle\left(A_{i} \wedge \bigwedge_{j} \neg B_{i j} \wedge \bigwedge_{k} \neg C_{i k}\right) \rightarrow[\beta]\langle\alpha\rangle \bigvee_{i}\left(A_{i} \wedge \bigwedge_{j} \neg B_{i j} \wedge \bigwedge_{k} \neg C_{i k}\right) .
$$

To establish this we observe that $\vdash \neg C_{i k} \rightarrow[\beta] \neg C_{i k}$ and use large part of our reasoning before:

$$
\begin{aligned}
\langle\alpha\rangle\left(A_{i} \wedge \bigwedge_{j} \neg B_{i j} \wedge \bigwedge_{k} \neg C_{i k}\right) & \rightarrow \bigwedge_{k} \neg C_{i k} \wedge\langle\alpha\rangle A_{i} \\
& \rightarrow \bigwedge_{k} \neg C_{i k} \wedge[\beta]\langle\alpha\rangle A_{i} \\
& \rightarrow \bigwedge_{k}[\beta] \neg C_{i k} \wedge[\beta]\langle\alpha\rangle A_{i} \\
& \rightarrow[\beta]\left(\bigwedge_{k} \neg C_{i k}\right) \wedge[\beta]\langle\alpha\rangle A_{i} \\
& \rightarrow[\beta]\left(\bigwedge_{k} \neg C_{i k}\right) \wedge[\beta]\langle\alpha\rangle\left(A_{i} \wedge \wedge_{j} \neg B_{i j}\right) \\
& \rightarrow[\beta]\left(\bigwedge_{k} \neg C_{i k} \wedge\langle\alpha\rangle\left(A_{i} \wedge \wedge_{j} \neg B_{i j}\right)\right) \\
& \rightarrow[\beta]\langle\alpha\rangle\left(A_{i} \wedge \bigwedge_{j} \neg B_{i j} \wedge \bigwedge_{k} \neg C_{i k}\right) \\
& \rightarrow[\beta]\langle\alpha\rangle \bigvee_{i}\left(A_{i} \wedge \wedge_{j} \neg B_{i j} \wedge \bigwedge_{k} \neg C_{i k}\right)
\end{aligned}
$$

Giving an explicit proof for the full version of Löb's axiom from the restricted ones seems to be rather involved thus we choose another proof strategy.

We observe that the only (!) application of Löb's axiom in this paper is in Lemma 5.6 where it is actually restricted to worms. Thus, with the restricted version of Löb's axiom we come to the same decision procedure and the same set of unique WNFs whence the two logics $w-G L P_{\Lambda}^{0}$ and $G L P_{\Lambda}^{0}$ prove the same set of theorems. 


\section{Acknowledgements}

The first author was supported by the Russian Foundation for Basic Research (RFBR), the Presidential council for support of leading scientific schools, and the Swiss-Russian cooperation project STCP-CH-RU "Computational proof theory".

The second author was supported by the project "Alternative interpretations of non-classical logics" (HUM-5844) of the Junta de Andalucía.

The third author wishes to thank the participants of the Seminari Cuc in Barcelona for feedback, question, suggestions and discussions: Joan Bagaria, Felix Bou, Ramon Jansana and Enrique Casanovas.

\section{References}

[1] L. D. Beklemishev and D. Gabelaia. Topological completeness of the provability logic GLP. ArXiv, 1106.5693v1 [math.LO], 2011. To appear in Annals of Pure and Applied Logic.

[2] L. D. Beklemishev, J. J. Joosten, and M. Vervoort. A finitary treatment of the closed fragment of Japaridze's provability logic. Journal of Logic and Computation, 15:447-463, 2005.

[3] L.D. Beklemishev. Provability algebras and proof-theoretic ordinals, I. Annals of Pure and Applied Logic, 128:103-124, 2004.

[4] L.D. Beklemishev. Veblen hierarchy in the context of provability algebras. In P. Hájek, L. Valdés-Villanueva, and D. Westerståhl, editors, Logic, Methodology and Philosophy of Science, Proceedings of the Twelfth International Congress, pages 65-78. Kings College Publications, 2005.

[5] L.D. Beklemishev. The Worm principle. In Z. Chatzidakis, P. Koepke, and W. Pohlers, editors, Logic Colloquium 2002, Lecture Notes in Logic 27, pages 75-95. ASL Publications, 2006.

[6] L.D. Beklemishev. Kripke semantics for provability logic GLP. Annals of Pure and Applied Logic, 161(6):737-744, 2010.

[7] L.D. Beklemishev. On the Craig interpolation and the fixed point properties of GLP. In S. Feferman et al., editor, Proofs, Categories and Computations. Essays in honor of G. Mints, Tributes, pages 49-60. 
College Publications, London, 2010. Preprint: Logic Group Preprint Series 262, University of Utrecht, Dec. 2007.

[8] L.D. Beklemishev. A simplified proof of the arithmetical completeness theorem for the provability logic GLP. Trudy Matematicheskogo Instituta imeni V.A. Steklova, 274(3):32-40, 2011. English translation: Proceedings of the Steklov Institute of Mathematics, 274(3):25-33, 2011.

[9] G. S. Boolos. The Logic of Provability. Cambridge University Press, Cambridge, 1993.

[10] Chagrov, A. V. and Rybakov, M. N. How many variables does one need to prove PSPACE-hardness of modal logics. In Advances in Modal Logic, volume 4, pages 71-82, 2003.

[11] S. Feferman and C. Spector. Incompleteness along paths in progressions of theories. The Journal of Symbolic Logic, 27:383-390, 1962.

[12] D. Fernández-Duque. The polytopologies of transfinite provability logic. ArXiv, 1207.6595 [math.LO], 2012.

[13] D. Fernández-Duque and J. J. Joosten. Models of transfinite provability logics. Journal of Symbolic Logic, 2012. Accepted for publication.

[14] Fernández-Duque, D. and Joosten, J. J. Hyperations, Veblen progressions and transfinite iteration of ordinal functions. Submitted, May 2012.

[15] Fernández-Duque, D. and Joosten, J. J. Kripke models of transfinite provability logic. In Advances in Modal Logic, volume 9, pages 185-199. College Publications, 2012.

[16] Fernández-Duque, D. and Joosten, J. J. Turing progressions and their well-orders. In How the world computes, Lecture Notes in Computer Science, pages 212-221. Springer, 2012.

[17] Fernández-Duque, D. and Joosten, J. J. Well-orders in the transfinite Japaridze algebra II. forthcoming, 2012.

[18] T. F. Icard III. Models of the polymodal provability logic. Master's thesis, Institute for Logic Language and Information, 2008.

[19] T. F. Icard III. A topological study of the closed fragment of GLP. Journal of Logic and Computation, 21:683-696, 2011. 
[20] K. N. Ignatiev. On strong provability predicates and the associated modal logics. The Journal of Symbolic Logic, 58:249-290, 1993.

[21] G.K. Japaridze. The modal logical means of investigation of provability. PhD thesis, Moscow State University, 1986. In Russian.

[22] J. J. Joosten. Intepretability Formalized. PhD thesis, Utrecht University, 2004.

[23] F. Pakhomov. On the complexity of the closed fragment of Japaridze's provability logic. In T. Bolander, T. Braüner, S. Ghilardi, and L. Moss, editors, 9-th Advances in Modal Logic, AiML 2012, Short Presentations, pages 56-59, 2012.

[24] D.S. Shamkanov. Interpolation properties of provability logics GL and GLP. Trudy Matematicheskogo Instituta imeni V.A. Steklova, 274(3):329-342, 2011. English translation: Proceedings of the Steklov Institute of Mathematics, 274(3):303-316, 2011. 